\title{
Effects of first-line chemotherapy on natural killer cells in adult T-cell leukemia-lymphoma and peripheral T-cell lymphoma
}

\author{
Michinori Ogura ${ }^{1,2} \cdot$ Takashi Ishida $^{3} \cdot$ Kunihiro Tsukasaki $^{4,5} \cdot$ Takeshi Takahashi $^{6}$. \\ Atae Utsunomiya ${ }^{7}$
}

Received: 7 January 2016 / Accepted: 2 June 2016 / Published online: 11 June 2016

(C) The Author(s) 2016. This article is published with open access at Springerlink.com

\begin{abstract}
Purpose Natural killer (NK) cells are well known to be the most important effector cells mediating antibody-dependent cellular cytotoxicity (ADCC) which is an important mechanism of action of antibody drugs. We evaluated the effects of chemotherapy on the cell number and activity of NK cells from patients who received the vincristine-cyclophosphamide-doxorubicin-prednisone (VCAP), doxorubicin-ranimustine-prednisone (AMP), and vindesine-etoposide-carboplatin-prednisone (VECP) (mLSG15) or mLSG15-like $(-\mathrm{L})$ regimen, which is one of the standard of cares for newly diagnosed adult T-cell leukemia-lymphoma (ATL), or the cyclophosphamide-doxorubicin-vincristine-prednisone (CHOP) or CHOP-L regimen which is another standard of care for ATL and peripheral T-cell lymphoma (PTCL).
\end{abstract}

Michinori Ogura

mi-ogura@naa.att.ne.jp

1 Department of Hematology, Tokai Central Hospital, Kakamigahara, Gifu 504-8601, Japan

2 Department of Hematology and Oncology, Nagoya Daini Red Cross Hospital, Nagoya, Aichi 466-8650, Japan

3 Department of Hematology and Oncology, Nagoya City University Graduate School of Medical Sciences, Nagoya, Aichi 467-8601, Japan

4 Department of Hematology, National Cancer Center Hospital East, Kashiwa, Chiba 277-8577, Japan

5 Department of Hematology and Molecular Medicine, Nagasaki University Graduate School of Biomedical Science, Nagasaki-shi, Nagasaki 852-8523, Japan

6 Oncology R\&D Unit, R\&D Division, Kyowa Hakko Kirin Co., Ltd., Chiyoda-ku, Tokyo 100-8185, Japan

7 Department of Hematology, Imamura Bun-in Hospital, Kamoikeshinmachi, Kagoshima 890-0064, Japan
Methods The number of lymphocytes and NK cells, and NK cell activity, were assessed using flow cytometry and a ${ }^{51} \mathrm{Cr}$ release assay, respectively.

Results A total of 26 patients with untreated ATL or PTCL were enrolled, and blood samples from 25 patients were evaluable. NK cell number in ATL decreased after mLSG15/-L treatment, and the degree of decrease in the NK cell number was more prominent just before VECP therapy (Day 15-17 of each cycle) than just before VCAP therapy (Day 1 of each cycle). The NK cell number in ATL after $\mathrm{CHOP} /$-L treatment also decreased. Interestingly, the NK cell activity showed a tendency to increase after the treatment. NK cell number in PTCL did not decrease by $\mathrm{CHOP} /$-L regimen, but the activity was slightly decreased after the treatment.

Conclusions These results indicate that the effects of chemotherapeutic agents on NK cells vary according to the disease type and intensity of chemotherapy.

Keywords Antibody · ATL · Chemotherapy · NK cell · PTCL

$\begin{array}{ll}\text { Abbreviations } \\ \text { ADCC } & \text { Antibody-dependent cellular cytotoxicity } \\ \text { AITL } & \text { Angioimmunoblastic T-cell lymphoma } \\ \text { ALCL } & \text { Anaplastic large cell lymphoma } \\ \text { ALK } & \text { Anaplastic lymphoma kinase } \\ \text { ATL } & \text { Adult T-cell leukemia-lymphoma } \\ \text { CCR4 } & \text { Chemokine receptor 4 } \\ \text { CD } & \text { Cluster of differentiation } \\ \text { CHOP } & \begin{array}{l}\text { Cyclophosphamide, doxorubicin, vincris- } \\ \text { tine, and prednisone }\end{array} \\ \text { JCOG } & \text { Japan Clinical Oncology Group } \\ \text { mLSG15 } & \text { VCAP-AMP-VECP; vincristine, cyclo- } \\ & \text { phosphamide, doxorubicin, and prednisone; }\end{array}$

Abbreviations

ADCC Antibody-dependent cellular cytotoxicity

AITL Angioimmunoblastic T-cell lymphoma

ALCL Anaplastic large cell lymphoma

ALK Anaplastic lymphoma kinase

ATL Adult T-cell leukemia-lymphoma

$\mathrm{CCR} 4$ Chemokine receptor 4

$\mathrm{CD} \quad$ Cluster of differentiation Cyclophosphamide, doxorubicin, vincristine, and prednisone

JCOG Japan Clinical Oncology Group phosphamide, doxorubicin, and prednisone; 
doxorubicin, ranimustine, and prednisone; and vindesine, etoposide, carboplatin, and prednisone

NK Natural killer

OS Overall survival

PTCL Peripheral T-cell lymphoma

PTCL-NOS Peripheral T-cell lymphoma, not otherwise specified

SSC-H Side scatter-height

\section{Introduction}

ATL belongs to the category of mature T/NK-cell neoplasms [1] and occurs secondary to HTLV-1 infection [24]. ATL frequently occurs in the HTLV-1 endemic area in Kyushu islands, the western part of Japan [5], and its prognosis is the worst among common subtypes of mature T/ NK-cell neoplasms [6]. Recently, VCAP-AMP-VECP regimen (mLSG15), which was a modified version of LSG15 [7], developed by the Japan Clinical Oncology Group (JCOG) has been established as a standard of care in newly diagnosed aggressive ATL [8]. However, the results are still not satisfactory, with a median survival of 13 months and a 3-year overall survival (OS) rate of only $24 \%$ [9]. Other mature T/NK-cell neoplasms include peripheral T-cell lymphoma, not otherwise specified (PTCL-NOS), angioimmunoblastic T-cell lymphoma (AITL), and anaplastic large cell lymphoma (ALCL). These disease subtypes are collectively called PTCL [1]. The current standard of care for PTCL is CHOP therapy including the CHOP-L regimen [10]; however, it has been reported that PTCL shows a generally poor prognosis, with 5-year OS rates of only $32 \%$ in PTCL-NOS and AITL, except for in anaplastic lymphoma kinase (ALK)-positive ALCL, which is associated with a 5-year OS rate of $70 \%$ [6].

In recent years, the efficacies of various therapeutic monoclonal antibody (mAb)-based monotherapies have been investigated in PTCL. These mAbs include the antiCD52 mAb alemtuzumab [11, 12], the anti-CC chemokine receptor 4 (CCR4) mAb mogamulizumab [13-15], and the anti-CD30 antibody drug conjugate mAb, brentuximab vedotin [16]. Based on evidence indicating that a combination of the anti-CD20 mAb rituximab [17], and chemotherapy including CHOP therapy achieved a marked improvement in therapeutic efficacy in patients with B-cell non-Hodgkin lymphomas, the combination of mAbs and chemotherapy is expected to represent a promising regimen for mature T/NK-cell neoplasms.

ADCC is an important tumor cell-killing mechanism of action of mAbs including the anti-CCR4 mAb mogamulizumab, which has been approved for CCR4-positive ATL and relapsed/refractory PTCL in Japan. Mogamulizumab is a defucosylated $\mathrm{mAb}$, resulting in highly enhanced ADCC by increased binding affinity to the Fc $\gamma$ III receptor (CD16) on effector cells [18-20]. It has been also shown that the ADCC of mogamulizumab mainly depends on the amount of effector cells such as CD16/CD56 double-positive NK cells [19]; however, to our knowledge, there have been no reports regarding the effects of various chemotherapeutic agents on CD16/CD56 double-positive NK cells cause high ADCC or other effector cells to date. In this regard, we carried out the present study to evaluate the effects of two first-line therapies, namely the mLSG15 or mLSG15L (mLSG15/-L) and CHOP or CHOP-L (CHOP/-L) regimens, on NK cell number and activity in newly diagnosed ATL and PTCL patients.

\section{Materials and methods}

\section{Study design}

This study was carried out in four institutions (Nagoya Daini Red Cross Hospital, Nagoya City University Hospital, Nagasaki University Hospital, and Imamura Bun-in Hospital) in collaboration with Kyowa Hakko Kirin Co., Ltd. (Tokyo, Japan) from May 2009 to November 2010. As per the study protocol, blood samples were collected at specified time points from patients with newly diagnosed ATL or PTCL receiving the $\mathrm{mLSG} 15 /-\mathrm{L}$ regimen or $\mathrm{CHOP} / \mathrm{L}$ regimen as the primary treatment. Cell numbers of lymphocytes and NK cells, and NK cell activity, were determined by flow cytometry, and ${ }^{51} \mathrm{Cr}$ release assay, respectively [21]. Blood samples were obtained at eight time points before the VCAP and VECP therapies in the first to the fourth cycle of the $\mathrm{mLSG} 15 /-\mathrm{L}$ regimen, and at eight points before the first to the eighth cycle of the CHOP/-L regimen. A blood sample of $10 \mathrm{~mL}$ was obtained at each time point, and the measurement was terminated when the treatment was discontinued or changed to another treatment.

Blood samples were also collected from healthy adult volunteers in a clinical research institution between November and December 2009, and their NK cell number and activity were measured in the same manner.

\section{Patients and healthy adult volunteers}

Patients with newly diagnosed and previously untreated ATL (acute, lymphoma, or unfavorable chronic type) or PTCL aged 20 years or older to whom it was planned to administer the $\mathrm{mLSG} 15 /-\mathrm{L}$ or $\mathrm{CHOP} / \mathrm{-L}$ regimen were eligible for the study. The unfavorable chronic type of ATL was defined by the presence of at least one of the following three factors: low serum albumin, high lactate dehydrogenase, or high blood urea nitrogen concentration [9]. 
The healthy adult volunteers were aged 40 or above who were negative for hepatitis B virus surface antigen, hepatitis $\mathrm{C}$ virus antibody, human immunodeficiency virus antibody, or syphilis test such as rapid plasma regain and treponema pallidum hemagglutination test. The blood test for HTLV-1 antibodies was not carried out for healthy adult volunteers. They had also no past history of allergic diseases such as atopic dermatitis, bronchial asthma, or hay fever. All patients and healthy adult volunteers signed written informed consent.

\section{Evaluation criteria and endpoint}

The immune status was evaluated in all eligible patients, and variations in NK cell number and activity before, during, and after chemotherapy were determined in order to speculate as to the usefulness of subsequent mAb therapy for these diseases.

The NK cell number was obtained through measurement by flow cytometry (3-color assay for CD45/CD16/CD56 in CD3-negative fraction). Figure 4 in "Appendix" shows the representative FACS plot defined by CD16 and CD56. The lymphocyte gate was determined by side scatter-height (SSC-H) and CD45. The gated cells were displayed on a plot of CD16 versus CD56 expression. The double-positive fraction of CD16/CD56 was defined as the NK cell. The NK cell number was calculated according to the following formula:

NK cell number $(/ \mu \mathrm{L})=$ lymphocyte count $(/ \mu \mathrm{L})$

$\times$ double-positive fraction of CD16/CD56 $(\%) \times 0.01$.

NK cell activity was obtained through measurement by ${ }^{51} \mathrm{Cr}$ release assay (an assay using the reaction of peripheral blood mononuclear cells (monocyte and lymphocyte) and ${ }^{51} \mathrm{Cr}$-labeled K562 cells at an effector/target ratio of 20:1), and was calculated according to the following formula:

NK cell activity $(\%)=$ Specific lysis $(\%)^{*}$

$\times$ double-positive fraction of CD16/CD56 $(\%) \times 0.01$

* $(\mathrm{E}-\mathrm{S}) /(\mathrm{M}-\mathrm{S}) \times 100$, where $E$ is the experimental release, $S$ is the spontaneous release, and $M$ is the maximum release.

\section{Statistical analysis}

Data were shown as box plots. For multiple comparison, Dwass, Steel, Critchlow-Fligner multiple comparison analysis was used as shown in Fig. 1. All statistical analyses were conducted by SAS ver 9.4 (SAS Institute Inc., Cary, NC, USA).

\section{Study oversight}

The study was sponsored by Kyowa Hakko Kirin Co., Ltd. The academic investigators and the sponsor were jointly responsible for the study design. The protocol was approved by the institutional review boards at each participating site, and the study was conducted complying with the ethical guidelines on clinical research and in accordance with the Declaration of Helsinki 1995. The blood sample assays using flow cytometry and ${ }^{51} \mathrm{Cr}$ release were outsourced to SRL Medisearch Inc. Data analysis was outsourced to Biostatistics center, Kurume university.

\section{Results}

\section{Patient characteristics}

The total number of patients enrolled was 26 , and 25 patients (14 patients with ATL and 11 patients with PTCL) were included in the data analysis. One patient was excluded from analysis due to a low initial lymphocyte count of $80 / \mu \mathrm{L}$. Data from this patient were rejected because it was judged to be inappropriate to use this value as the basis for examination of variations, and calculation of the NK cell number and activity. Table 1 shows the

Table 1 Patient demographics and clinical characteristics

\begin{tabular}{|c|c|c|c|c|c|}
\hline \multicolumn{2}{|c|}{ Characteristic } & \multicolumn{4}{|c|}{$\begin{array}{l}\text { ATL patients }(n=14) \text { PTCL patients } \\
\qquad(n=11)\end{array}$} \\
\hline & & \multicolumn{2}{|l|}{$n(\%)$} & \multicolumn{2}{|l|}{$n(\%)$} \\
\hline \multirow[t]{2}{*}{ Age (years) } & Median & 56 & & 69 & \\
\hline & Range & $47-72$ & & $36-78$ & \\
\hline \multirow[t]{2}{*}{ Sex } & Male & $9(64)$ & & $9(82)$ & \\
\hline & Female & $5(36)$ & & $2(18)$ & \\
\hline \multirow[t]{5}{*}{ ECOG PS } & 0 & $2(14)$ & & $5(45)$ & \\
\hline & 1 & $9(64)$ & & $4(36)$ & \\
\hline & 2 & $2(14)$ & & $2(18)$ & \\
\hline & 3 & $0(0)$ & & $0(0)$ & \\
\hline & 4 & $1(7)$ & & $0(0)$ & \\
\hline \multirow[t]{2}{*}{ Therapy } & CHOP/-L & $5(36)$ & & $11(100)$ & \\
\hline & mLSG15/-L & $9(64)$ & & $0(0)$ & \\
\hline \multirow[t]{5}{*}{ Subtype } & & Acute & $10(71)$ & AITL & $5(45)$ \\
\hline & & Lymphoma & $1(7)$ & PTCL-NOS & $3(27)$ \\
\hline & & Chronic & $3(21)$ & ALCL ALK- & $1(9)$ \\
\hline & & & & EATL & $1(9)$ \\
\hline & & & & SPTCL & $1(9)$ \\
\hline
\end{tabular}

AITL angioimmunoblastic T-cell lymphoma, ALCL anaplastic large cell lymphoma, $A L K$ anaplastic lymphoma kinase, $A T L$ adult T-cell leukemia-lymphoma, $C H O P$ cyclophosphamide, doxorubicin, vincristine, and prednisone, CHOP/-L CHOP or CHOP-like regimen, EATL enteropathy-associated T-cell lymphoma, ECOG Eastern Cooperative Oncology Group, $m L S G 15$ vincristine, cyclophosphamide, doxorubicin, prednisone and doxorubicin, ranimustine, prednisone and vindesine, etoposide, carboplatin, prednisone, $m L S G 15 /-L$ mLSG15 or mLSG15-like regimen, NOS not otherwise specified, $P S$ performance status, $P T C L$ peripheral T-cell lymphoma, SPTCL subcutaneous panniculitis-like T-cell lymphoma 
demographics and clinical characteristics of the 25 analyzed patients, and Table 2 shows the breakdown of patients on chemotherapy in relation to the disease subtype. The mLSG15/-L regimen was administered to $9(64 \%)$ patients with ATL. It should be noted that although the number of patients analyzed was limited, no marked difference was found in disease subtype according to the type of chemotherapy (mLSG15/-L vs. CHOP/-L). The CHOP/-L regimen was administered to all (100\%) patients with PTCL.

Table 3 in "Appendix" shows the breakdown of ATL patients received with $\mathrm{mLSG} 15 /-\mathrm{L}$ regimen and $\mathrm{CHOP} / \mathrm{L}$ regimen, and Table 4 in "Appendix" shows the breakdown of PTCL patients received with CHOP/-L regimen. Disease progressions were almost reasons for taken off these therapies. None of ATL patients received both $\mathrm{mLSG} 15 /-\mathrm{L}$ and $\mathrm{CHOP} / \mathrm{L}$ regimens.

\section{Lymphocyte counts and NK cell number and activity before treatment initiation}

Figure 1 shows the lymphocyte count, NK cell number, and NK cell activity determined in 14 patients with ATL, 11 patients with PTCL, and 10 healthy adult volunteers. The lymphocyte count before initiation of treatment was significantly higher by $1 \log$ in ATL compared to in PTCL patients (Fig. 1a), which was probably attributable to the fact that patients with acute-type ATL who had ATL cells in the peripheral blood [22] accounted for $71 \%$ of the patients with
ATL (Table 2). There was no significant difference in the number of CD16/CD56 double-positive NK cells which can contribute to ADCC of mogamulizumab, before treatment initiation between ATL and PTCL patients, or between these patients and healthy adult volunteers (Fig. 1b). Although the NK cell activity before treatment initiation was markedly lower in ATL than in PTCL patients (Fig. 1c), there was no significant difference in the NK cell activity between patients with PTCL and healthy adult volunteers (Fig. 1c).

\section{Variations in the NK cell number and activity in ATL patients after treatment initiation}

The NK cell number in patients with ATL was decreased as a result of the mLSG15/-L regimen, and this decrease was more prominent before VECP therapy (Day 15-17 of each cycle) than before VCAP therapy (Day 1 of each cycle) (Fig. 2a). The NK cell number in patients with ATL also decreased by the CHOP/-L regimen (Fig. 2c). However, the NK cell activity by the CHOP/-L regimen showed a tendency to increase (Fig. 2d), although there was not marked difference by mLSG15/-L regimen (Fig. 2b).

\section{Variations in NK cell number and activity in PTCL patients after treatment initiation}

In PTCL patients, the NK cell number did not decrease by the CHOP/-L regimen (Fig. 3a), though the NK cell
Table 2 Breakdown of patients on chemotherapy in relation to the disease subtype

\begin{tabular}{|c|c|c|c|}
\hline \multirow[t]{2}{*}{ ATL subtype $(n=14)$} & \multirow[t]{2}{*}{$n(\%)$} & \multicolumn{2}{|l|}{ Therapy } \\
\hline & & $\begin{array}{l}\text { mLSG15/-L } \\
n(\%)\end{array}$ & $\begin{array}{l}\mathrm{CHOP} /-\mathrm{L}^{\mathrm{a}} \\
n(\%)\end{array}$ \\
\hline Acute & $10(71)$ & $6(43)$ & $4(29)$ \\
\hline Lymphoma & $1(7)$ & $1(7)$ & $0(0)$ \\
\hline Chronic & $3(21)$ & $2(14)$ & $1(7)$ \\
\hline \multirow[t]{2}{*}{ PTCL subtype $(n=11)$} & \multirow[t]{2}{*}{$n(\%)$} & \multicolumn{2}{|l|}{ Therapy } \\
\hline & & $\begin{array}{l}\text { CHOP } \\
n(\%)\end{array}$ & $\begin{array}{l}\text { CHOP-L }{ }^{\mathrm{a}} \\
n(\%)\end{array}$ \\
\hline AITL & $5(45)$ & $1(9)$ & $4(36)$ \\
\hline PTCL-NOS & $3(27)$ & $1(9)$ & $2(18)$ \\
\hline ALCL ALK- & $1(9)$ & $1(9)$ & $0(0)$ \\
\hline EATL & $1(9)$ & $0(0)$ & $1(9)$ \\
\hline SPTCL & $1(9)$ & $0(0)$ & $1(9)$ \\
\hline
\end{tabular}

AITL angioimmunoblastic T-cell lymphoma, ALCL anaplastic large cell lymphoma, ALK anaplastic lymphoma kinase, $A T L$ adult T-cell leukemia-lymphoma, $C H O P$ cyclophosphamide, doxorubicin, vincristine, and prednisone; $C H O P-L$ CHOP-like regimen, $C H O P /-L$ CHOP or CHOP-like regimen, EATL enteropathy-associated T-cell lymphoma, $m L S G 15$ vincristine, cyclophosphamide, doxorubicin, prednisone and doxorubicin, ranimustine, prednisone and vindesine, etoposide, carboplatin, prednisone, $m L S G 15 /$ $L$ mLSG15 or mLSG15-like regimen, NOS not otherwise specified, PTCL peripheral T-cell lymphoma, SPTCL subcutaneous panniculitis-like T-cell lymphoma

a THP-COP, vincristine, pirarubicin, cyclophosphamide, and prednisone 
$/ \mu \mathrm{L}$

(a) Lymphocyte count

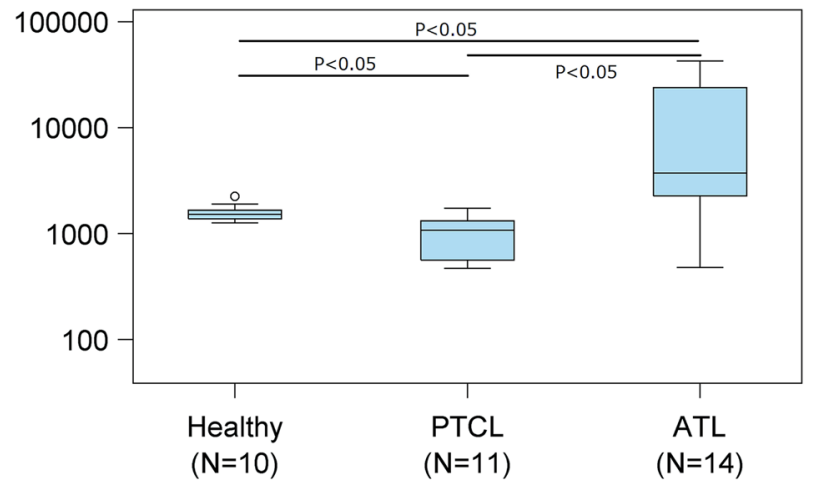

$/ \mu \mathrm{L} \quad$ (b) NK cell number
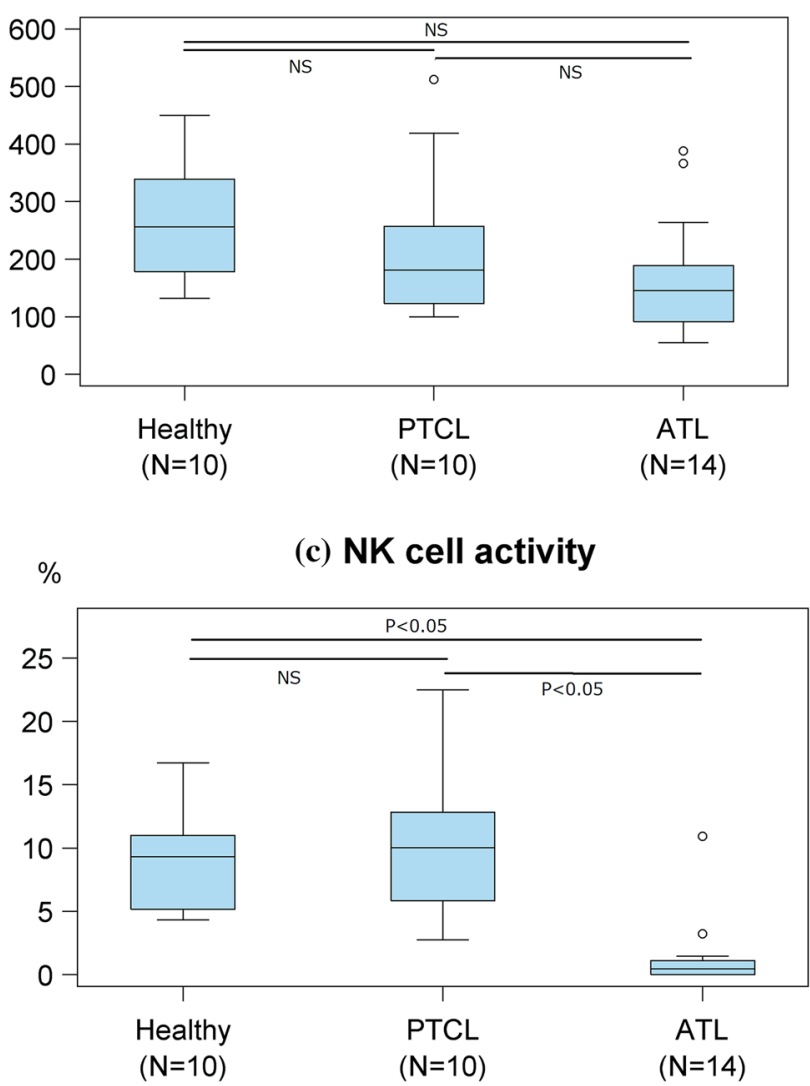

Fig. 1 Lymphocyte count, natural killer (NK) cell number, and NK cell activity before treatment initiation as determined using flow cytometry (cell number) and a ${ }^{51} \mathrm{Cr}$ release assay (activity). a The mean lymphocyte count in healthy volunteers, peripheral T-cell lymphoma (PTCL) patients, and adult T-cell leukemia-lymphoma (ATL) patients were 1580,991 , and $11,618 / \mu \mathrm{L}$, respectively. b The NK cell number in healthy volunteers, PTCL patients, and ATL patients, the corresponding mean values were 262,224 , and $166 / \mu \mathrm{L}$, respectively. c The NK cell activity values in healthy volunteers, PTCL patients, and ATL patients, the corresponding mean values were $8.8,10.4$, and $1.4 \%$, respectively number in patients with ATL decreased by this regimen (Fig. 2c). The NK cell activity in PTCL patients slightly decreased by the CHOP/-L regimen (Fig. 3b).

\section{Discussion}

In this study, the effects of first-line therapy for newly diagnosed ATL and PTCL patients on NK cells defined as CD16/CD56 double-positive ones can cause high ADCC for defucosylated antibodies such as mogamulizumab were evaluated using NK cell number and NK cell activity substitute functional cell vitality as indices. These data showed that the effects of chemotherapy on NK cells varied according to the disease (ATL or PTCL) and the regimen used and/or the number of courses of chemotherapy (mLSG15/-L regimen or CHOP/-L regimen). Furthermore, it was also found that the NK cell activity before treatment initiation was markedly lower in ATL than in PTCL patients. This low activity of NK cell in ATL may be caused by suppression of NK cells by a subset of ATL cells functioning as Treg cells [23-25]. These results suggest that it is more difficult for some therapeutic mAbs that require an effector function to exert their therapeutic effects in ATL compared to in PTCL, although the mechanism of NK cell activity may be different from that of ADCC. However, it cannot be directly concluded that therapeutic Abs are less likely to exert benefits in patients with ATL. In fact, the NK cell number in cases treated with the mLSG15/-L regimen tended to be restored before the initiation of each chemotherapeutic cycle (VCAP therapy) in comparison with before toward the end of each cycle (VECP therapy). This finding suggests that in cases where mAbs are combined with the $\mathrm{mLSG} 15 /-\mathrm{L}$ regimen, some therapeutic mAbs may be more likely to exert their beneficial effects when they are used before the initiation of each cycle. Additionally, the NK cell activity tended to increase after the CHOP/-L regimen than after the mLSG15/-L regimen in patients with previously untreated ATL. Therapeutic effect of the administered chemotherapy against more suppressive ATL cells compared to PTCL may result in increased vitality per cell of survived NK cells in ATL patients. However, the details of why CHOP/-L treatment tended to produce better results compared to those of more potent intensive regimen, mLSG15/-L, are necessary to carefully consider, while paying heed to direct effects on NK cells by each chemotherapy itself. Therefore, it is not possible to determine which of the two regimens, both of which consist of drugs with different solitary effects, should be recommended as the most suitable 
(a) NK cell number (mLSG15/-L,ATL)

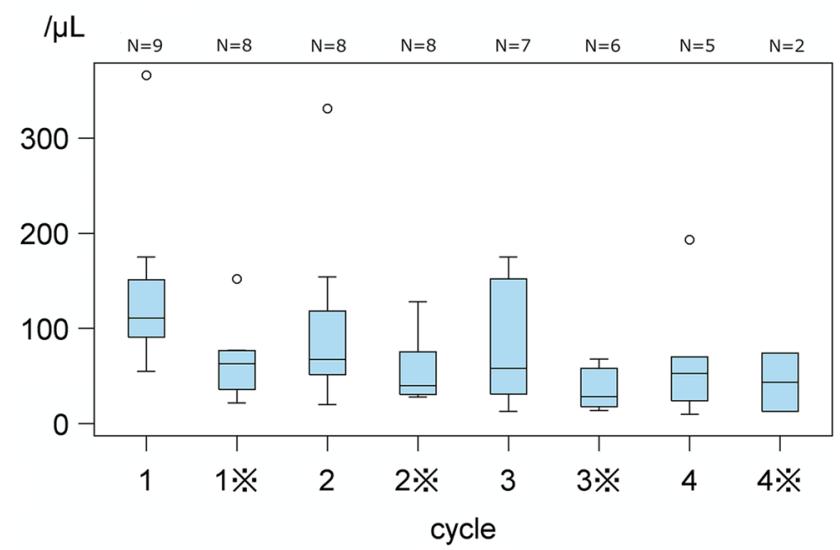

(b) NK cell activity (mLSG15/-L,ATL)

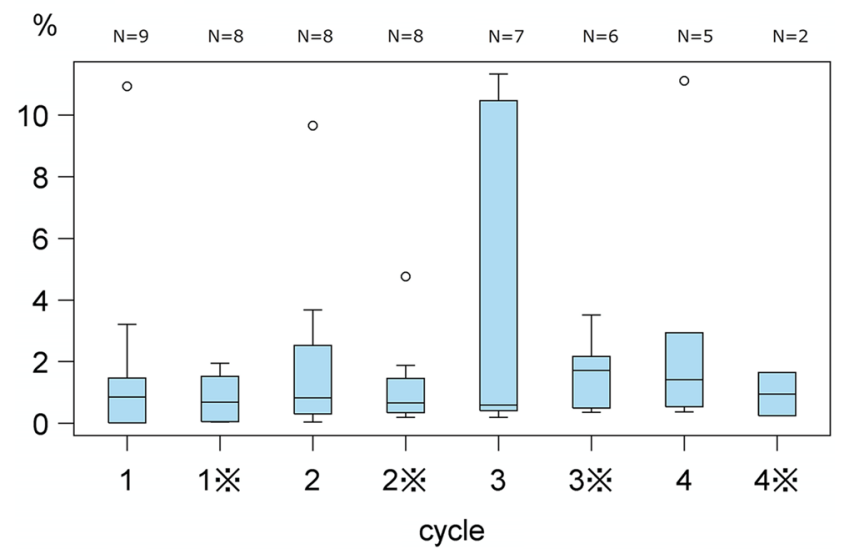

Fig. 2 Variations in natural killer (NK) cell number and NK cell activity in adult T-cell leukemia-lymphoma (ATL) patients. a NK cell number determined before (Pre) administration at VCAP (Day 1 of each cycle) and VECP (Day 15-17 of each cycle: ※) of mLSG15/$\mathrm{L}$ treatment initiation. b NK cell activity determined before (Pre)

chemotherapy to be combined with therapeutic Abs. However, the CHOP/-L regimen, which was associated with a less prominent decrease in totally NK cell activity, seems to be more promising, assuming that patients respond similarly to chemotherapy alone. Further investigations in a greater number of patients are needed, because this was a small-scale study.

Recently, it was carried out a phase II controlled clinical study (clinical trial information: NCT01173887) to examine the effects of combination treatment using the mLSG15 regimen and mogamulizumab in patients with untreated ATL [26]. In that phase II study, mogamulizumab was administered at intervals of 2 weeks (at the same time as VCAP and VECP administration) over four courses of the mLSG15 regimen ( 8 times in total), i.e., at the same times as the blood sampling was performed in the present study. The results showed that the complete response rate and overall response rate in the 29 (c) NK cell number (CHOP/-L,ATL)

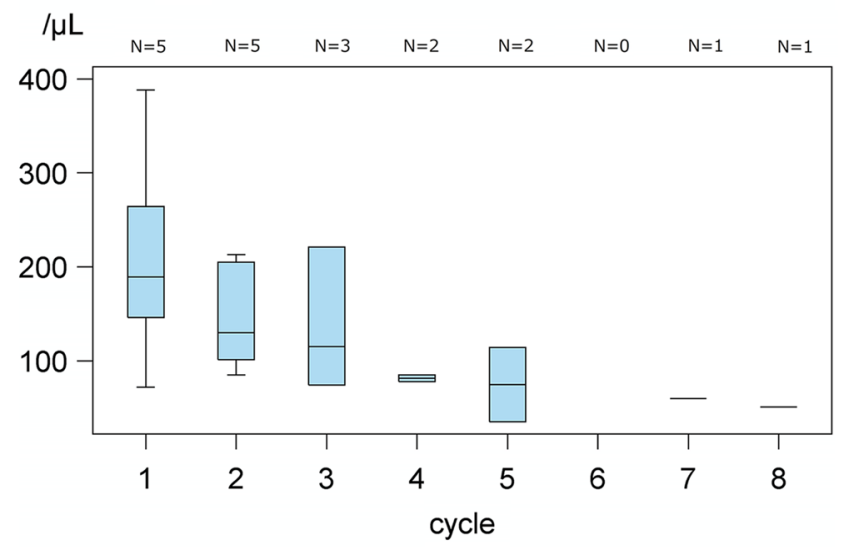

(d) NK cell activity (CHOP/-L,ATL)

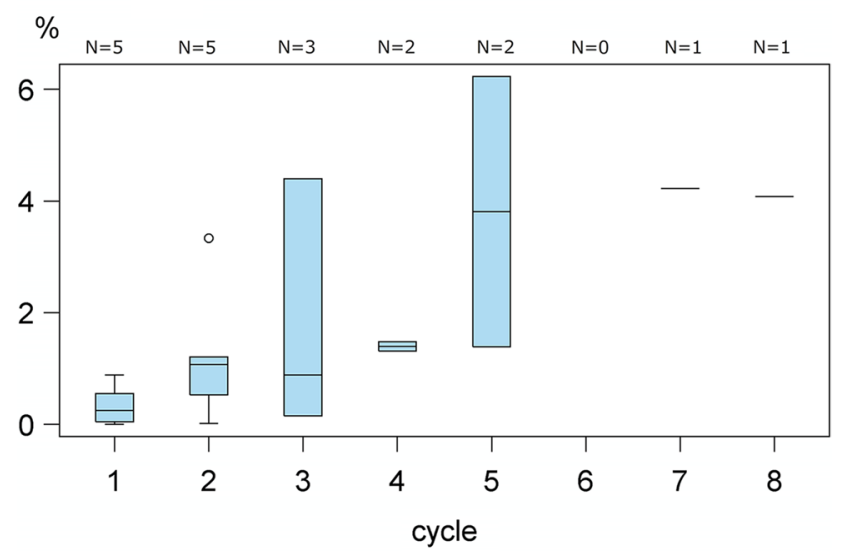

administration at VCAP (Day 1 of each cycle) and VECP (Day 15-17 of each cycle: $※)$ of $\mathrm{mLSG} 15 /-\mathrm{L}$ treatment initiation. $\mathbf{c}$ NK cell number determined before (Pre) administration at each cycle of $\mathrm{CHOP} /$ $\mathrm{L}$ treatment initiation. d NK cell activity determined before (Pre) administration at each cycle of $\mathrm{CHOP} / \mathrm{-L}$ treatment initiation

patients administered mLSG15 + mogamulizumab versus the 24 patients administered the mLSG15 alone were 52 versus $33 \%$ and 86 versus $75 \%$, respectively, with mLSG15 combined with mogamulizumab showing better efficacy in both indices. These data indicated that combining mogamulizumab with mLSG15 showed an additive effect with mLSG15, which tended to cause a greater decrease in NK cell number than the CHOP/-L regimen. Therefore, we speculate that mogamulizumab combination with the CHOP/-L regimen could also be beneficial for patients in whom the mLSG15 regimen is difficult to implement because of advanced age or for other reasons. Further investigations are needed to examine this presumption.

On the other hand, in patients with PTCL, the decrease in NK cell number and activity after the standard chemotherapy, i.e., the $\mathrm{CHOP} / \mathrm{L}$ regimen, was relatively small, as compared to the CHOP/-L treated patients with ATL, who 
(a) NK cell number (CHOP/-L,PTCL)

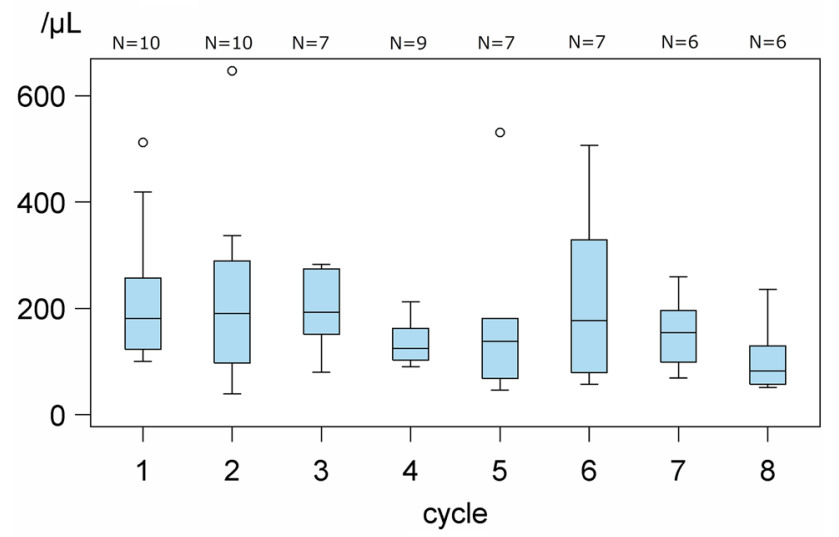

(b) NK cell activity (CHOP/-L,PTCL)

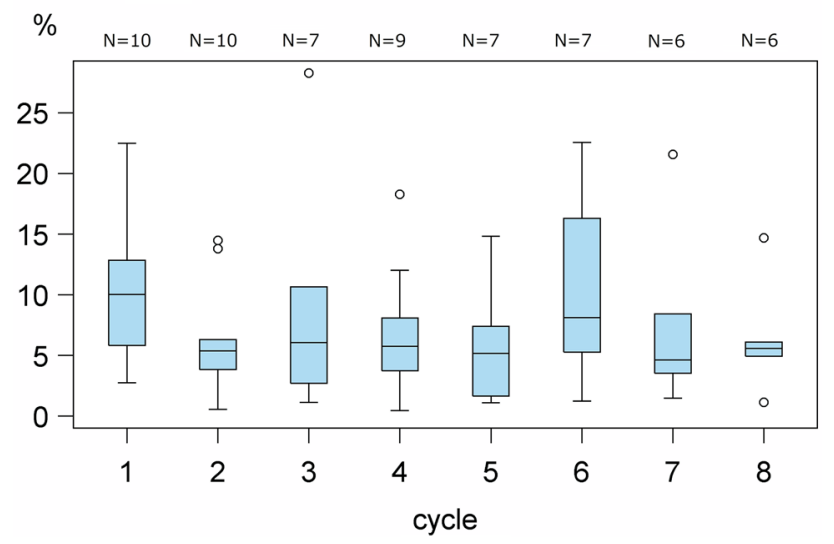

Fig. 3 Variations in natural killer (NK) cell number and NK cell activity in peripheral T-cell lymphoma (PTCL) patients. a NK cell number determined before (Pre) administration at each cycle of $\mathrm{CHOP} / \mathrm{L}$ treatment initiation. b NK cell activity determined before (Pre) administration at each cycle of $\mathrm{CHOP} /$-L treatment initiation

would be immune suppressive condition as seen in Fig. 1c which shows significant lower activity of NK cells in ATL patients compared to PTCL. This result suggests that the effect of combining therapeutic Abs with chemotherapy is considerably more promising in PTCL patients. Indeed, a phase II clinical study of alemtuzumab in combination with CHOP demonstrated favorable effects, with complete response and overall response rates in 24 patients of 71 and $75 \%$, respectively [27]. The efficacy of mogamulizumab in combination with CHOP in patients with untreated primary PTCL is also expected.

In conclusion, the results of the present study provide an important basis for future investigations of the combined effects of chemotherapy and therapeutic Abs [18-20] whose activity is affected by CD16/CD56 double-positive NK cells. Further analysis of CD16-positive other effector cells which antibodies bind also remains to be explored to fully understand the combination effects.

Acknowledgments We thank all the patients and their families, as well as the investigators who participated in this multicenter collaborative study.

\section{Compliance with ethical standards}

Conflict of interest Takeshi Takahashi is employee of Kyowa Hakko Kirin Co., Ltd.; Michinori Ogura serves as a consultant/advisory role to Mundipharma K.K., Meiji Seika Pharma Co., Ltd. and Celgene K.K.; Takashi Ishida and Atae Utsunomiya received honoraria from Kyowa Hakko Kirin Co., Ltd.; Takashi Ishida received research funding from Kyowa Hakko Kirin Co., Ltd., Bayer Pharma AG, and Celgene K.K.; Kunihiro Tsukasaki received research funding from Kyowa Hakko Kirin Co., Ltd.

Open Access This article is distributed under the terms of the Creative Commons Attribution 4.0 International License (http://creativecommons.org/licenses/by/4.0/), which permits unrestricted use, distribution, and reproduction in any medium, provided you give appropriate credit to the original author(s) and the source, provide a link to the Creative Commons license, and indicate if changes were made.

\section{Appendix}

See Tables 3, 4 and Fig. 4. 
Table 3 Breakdown of ATL patients received with (a) VCAP (Day 1 of each cycle) and VECP (Day $15-17$ of each cycle: $※$ ) of mLSG15/-L regimen, (b) $\mathrm{CHOP} / \mathrm{L}$ regimen
Table 4 Breakdown of PTCL patients received with $\mathrm{CHOP} /-\mathrm{L}$ regimen

Fig. 4 Representative FACS plot defined by CD16 and CD56. a Isotype control and b representative sample for ATL patients

\begin{tabular}{|c|c|c|c|c|c|c|c|c|}
\hline & \multicolumn{8}{|c|}{ Cycle number } \\
\hline & 1 & $1 ※$ & 2 & $2 ※$ & 3 & $3 ※$ & 4 & $4 ※$ \\
\hline \multicolumn{9}{|l|}{ (a) } \\
\hline Number of received patients & 9 & 8 & 8 & 8 & 7 & 6 & 5 & 2 \\
\hline Number of analyzed patients & 9 & 8 & 8 & 8 & 7 & 6 & 5 & 2 \\
\hline Number of missing data & - & 1 & 0 & 0 & 0 & 0 & 0 & 0 \\
\hline \multirow[t]{3}{*}{ Number of taken off therapy } & - & 0 & 1 & 1 & 2 & 3 & 4 & 7 \\
\hline & \multicolumn{8}{|c|}{ Cycle number } \\
\hline & 1 & 2 & 3 & 4 & 5 & 6 & 7 & 8 \\
\hline \multicolumn{9}{|l|}{ (b) } \\
\hline Number of received patients & 5 & 5 & 3 & 2 & 2 & 0 & 1 & 1 \\
\hline Number of analyzed patients & 5 & 5 & 3 & 2 & 2 & 0 & 1 & 1 \\
\hline Number of missing data & - & 0 & 0 & 0 & 0 & 2 & 0 & 0 \\
\hline Number of taken off therapy & - & 0 & 2 & 3 & 3 & 3 & 4 & 4 \\
\hline
\end{tabular}

\begin{tabular}{lllllllll}
\hline & \multicolumn{2}{l}{ Cycle number } & & & & \\
\cline { 2 - 9 } & 1 & 2 & 3 & 4 & 5 & 6 & 7 & 8 \\
\hline Number of received patients & 11 & 11 & 8 & 9 & 7 & 7 & 6 & 6 \\
Number of analyzed patients & 10 & 10 & 7 & 9 & 7 & 7 & 6 & 6 \\
Number of missing data & - & 0 & 2 & 0 & 1 & 1 & 1 & 0 \\
Number of taken off therapy & - & 0 & 1 & 1 & 2 & 2 & 3 & 4 \\
\hline
\end{tabular}

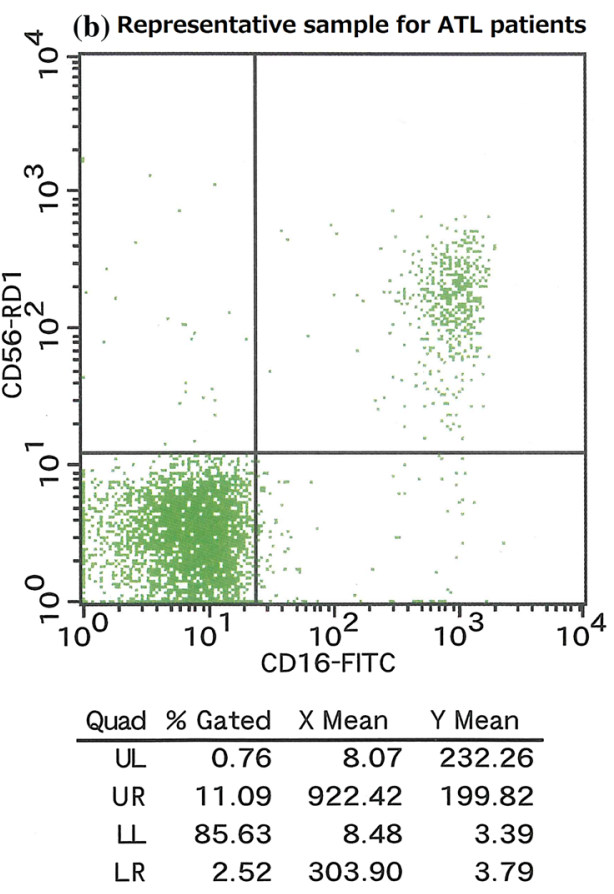




\section{References}

1. The International Agency for Research on Cancer (2008) WHO classification of tumors of hematopoietic and lymphoid tissues, 4th edn. International Agency for Research on Cancer (IARC), Lyon

2. Uchiyama T, Yodoi J, Sagawa K et al (1977) Adult T-cell leukemia: clinical and hematologic features of 16 cases. Blood 50:481-492

3. Poiesz BJ, Ruscetti FW, Gazdar AF et al (1980) Detection and isolation of type $\mathrm{C}$ retrovirus particles from fresh and cultured lymphocytes of a patient with cutaneous T-cell lymphoma. Proc Natl Acad Sci USA 77:7415-7419

4. Hinuma Y, Nagata K, Hanaoka M et al (1981) Adult T-cell leukemia: antigen in an ATL cell line and detection of antibodies to the antigen in human sera. Proc Natl Acad Sci USA 78:6476-6480

5. Chihara D, Ito H, Matsuda $T$ et al (2014) Differences in incidence and trends of haematological malignancies in Japan and the United States. Br J Haematol 164:536-545

6. Vose J, Armitage J, Weisenburger D (2008) International T-cell Lymphoma project. International peripheral T-cell and natural killer/T-cell lymphoma study: pathology findings and clinical outcomes. J Clin Oncol 26:4124-4130

7. Yamada Y, Tomonaga M, Fukuda H et al (2001) A new G-CSFsupported combination chemotherapy, LSG15, for adult T-cell leukaemia-lymphoma: Japan clinical oncology group study 9303. Br J Haematol 113:375-382

8. Tsukasaki K, Tobinai K, Hotta T et al (2012) Lymphoma study group of JCOG. Jpn J Clin Oncol 42:85-95

9. Tsukasaki K, Utsunomiya A, Fukuda H et al (2007) VCAPAMP-VECP compared with biweekly CHOP for adult T-cell leukemia-lymphoma: Japan Clinical Oncology Group Study JCOG9801. J Clin Oncol 25:5458-5464

10. National Comprehensive Cancer Network (2014) NCCN Clinical practice guidelines in oncology (NCCN Guidelines $\left.{ }^{\circledR}\right)$. NonHodgkin's lymphomas, version 2

11. Enblad G, Hagberg H, Erlanson M et al (2004) A pilot study of alemtuzumab (anti-CD52 monoclonal antibody) therapy for patients with relapsed or chemotherapy-refractory peripheral T-cell lymphomas. Blood 103:2920-2924

12. Zinzani PL, Alinari L, Tani M et al (2005) Preliminary observation of a phase II study of reduced-dose alemtuzumab in patients with pretreated T-cell lymphoma. Haematologica 90:702-703

13. Yamamoto K, Utsunomiya A, Tobinai K et al (2010) Phase I study of KW-0761, a defucosylated humanized anti-CCR4 antibody, in relapsed patients with adult T-cell leukemia-lymphoma and peripheral T-cell lymphoma. J Clin Oncol 28:1591-1598

14. Ishida $\mathrm{T}$, Joh $\mathrm{T}$, Uike $\mathrm{N}$ et al (2012) Defucosylated anti-CCR4 monoclonal antibody (KW-0761) for relapsed adult T-cell leukemia-lymphoma: a multicenter phase II study. J Clin Oncol 30:837-842

15. Ogura M, Ishida T, Hatake K et al (2014) Multicenter phase II study of mogamulizumab (KW-0761) a defucosylated anti-CC chemokine receptor 4 antibody, in patients with relapsed peripheral T-cell lymphoma and cutaneous T-cell lymphoma. J Clin Oncol 32:1157-1163

16. Pro B, Advani R, Brice P et al (2012) Brentuximab vedotin (SGN-35) in patients with relapsed or refractory systemic anaplastic large-cell lymphoma: results of a phase II study. J Clin Oncol 30:2190-2196

17. Coiffier B, Lepage E, Briere J et al (2002) CHOP chemotherapy plus rituximab compared with $\mathrm{CHOP}$ alone in elderly patients with diffuse large-B-cell lymphoma. N Engl J Med 346:235-242

18. Niwa R, Shoji-Hosaka E, Sakurada M et al (2004) Defucosylated chimeric anti-CC chemokine receptor 4 IgG1 with enhanced antibody-dependent cellular cytotoxicity shows potent therapeutic activity to T-cell leukemia and lymphoma. Cancer Res 64:2127-2133

19. Ishii T, Ishida T, Utsunomiya A et al (2010) Defucosylated humanized anti-CCR4 monoclonal antibody KW-0761 as a novel immunotherapeutic agent for adult T-cell leukemia/lymphoma. Clin Cancer Res 16:1520-1531

20. Yamane-Ohnuki N, Kinoshita S, Inoue-Urakubo M et al (2004) Establishment of FUT8 knockout Chinese hamster ovary cells: an ideal host cell line for producing completely defucosylated antibodies with enhanced antibody-dependent cellular cytotoxicity. Biotechnol Bioeng 87:614-622

21. Seto M, Takahashi T, Nakamura S et al (1983) In vivo antitumor effects of monoclonal antibodies with different immunoglobulin classes. Cancer Res 43:4768-4773

22. Shimoyama M (1991) Diagnostic criteria and classification of clinical subtypes of adult T-cell leukaemia lymphoma: a report from the Lymphoma Study Group (1984-87). Br J Haematol 79:428-437

23. Yano H, Ishida T, Inagaki A et al (2007) Regulatory T-cell function of adult T-cell leukemia/lymphoma cells. Int J Cancer 120:2052-2057

24. Trzonkowski P, Szmit E, Myśliwska J et al (2004) CD4 $4^{+} \mathrm{CD} 25^{+}$ $\mathrm{T}$ regulatory cells inhibit cytotoxic activity of $\mathrm{T} \mathrm{CD} 8^{+}$and $\mathrm{NK}$ lymphocytes in the direct cell-to-cell interaction. Clin Immunol 112:258-267

25. Smyth MJ, Teng MW, Swann J et al (2006) $\mathrm{CD} 4{ }^{+} \mathrm{CD} 25^{+} \mathrm{T}$ regulatory cells suppress NK cell-mediated immunotherapy of cancer. J Immunol 176:1582-1587

26. Ishida T, Jo T, Takemoto $\mathrm{S}$ et al (2015) Dose-intensified chemotherapy alone or in combination with mogamulizumab in newly diagnosed aggressive adult T-cell leukaemia-lymphoma: a randomized phase II study. Br J Haematol 169:672-682

27. Gallamini A, Zaja F, Patti C et al (2007) Alemtuzumab (Campath-1H) and CHOP chemotherapy as first-line treatment of peripheral T-cell lymphoma: results of a GITIL (Gruppo Italiano Terapie Innovative nei Linfomi) prospective multicenter trial. Blood 110:2316-2323 